\title{
Número de folhas associado com duplo anel e espigueta terminal em cultivares de trigo ${ }^{1}$
}

\author{
Leaf number associated with double ridge and terminal spikelet in wheat cultivars
}

\author{
Alencar Junior Zanon², Nereu Augusto Streck ${ }^{3 *}$, Hamilton Telles Rosa², Lidiane Cristine Walter ${ }^{4}$ e Cleber Maus \\ Alberto ${ }^{5}$
}

\begin{abstract}
RESUMO - O objetivo deste trabalho foi determinar o número de folhas, representado pelo Estágio de Haun, no duplo anel e na espigueta terminal de cultivares de trigo com diferentes ciclos de desenvolvimento e cultivados em diferentes datas de semeadura. Um experimento de campo foi realizado em Santa Maria, RS, com 13 datas de semeadura ao longo de três anos (2005; 2006 e 2007). Foram utilizados seis genótipos de trigo com ciclos de desenvolvimento variando de precoce a tardio: BRS Louro, CEP 52, BRS 177, CEP 51, Nova Era e BRS Tarumã. O delineamento experimental utilizado foi blocos completos casualizados, com seis tratamentos (cultivares) e quatro repetições. Foram determinados os estágios início do perfilhamento, duplo anel (DA), espigueta terminal (ET) e o número final de folhas no colmo principal (NFF) e na data em que ocorreu o DA e a ET foi quantificado o número de folhas totalmente expandidas mais a fração decimal do comprimento da lâmina foliar da última folha (em expansão) em relação à penúltima folha (expandida), com os quais foi calculado o Estágio de Haun (HS). Os resultados indicam que o número de folhas no colmo principal, representado pelo HS, no duplo anel $\left(\mathrm{HS}_{\mathrm{DA}}\right.$ ) e na espigueta terminal ( $\mathrm{HS}_{\mathrm{ET}}$ ) em trigo, varia com a cultivar (grupo de maturação) e com a data de semeadura. Práticas de manejo como a adubação nitrogenada e a aplicação de herbicidas hormonais podem ser melhoradas usando-se o $\mathrm{HS}_{\mathrm{DA}}$ e $\mathrm{HS}_{\mathrm{ET}}$ ao invés do início do perfilhamento e elongação do colmo, respectivamente.
\end{abstract}

Palavras-chave: Trigo-adubação nitrogenada. Semeadura. Herbicidas.

\begin{abstract}
The objective of this study was to determine the number of leaves, represented by the Haun stage, in the double ridge and terminal spikelet of wheat cultivars with different development cycles and grown on different sowing dates. A field experiment was conducted in Santa Maria, RS with 13 sowing dates over three years (2005, 2006 and 2007). Six wheat genotypes were used with development cycles ranging from early to late: BRS Louro, CEP 52, BRS 177, CEP 51, Nova Era and BRS Tarumã. The experimental design used was of randomized complete blocks, with six treatments (cultivars) and four repetitions. The early stages of tillering, double-ridging (DA), terminal spikelet (ET) and the final number of leaves on the main stem (NFF) were determined, and on the date of DA and ET, the number of fully expanded leaves was quantified together with the decimal fraction of the length of the leaf blade of the last leaf (unfolded) in relation to the penultimate leaf (unfolding), with which the Haun Stage (HS) was calculated. The results indicate that the number of leaves on the main stem, represented by $\mathrm{HS}$, in the double ridge $\left(\mathrm{HS}_{\mathrm{DA}}\right)$ and in the terminal spikelet $\left(\mathrm{HS}_{\mathrm{ET}}\right)$ in wheat, varies with the cultivar (maturity group) and the date of sowing. Management practices such as fertilization with nitrogen and the application of hormonal herbicides can be improved by using the $\mathrm{HS}_{\mathrm{DA}}$ and $\mathrm{HS}_{\mathrm{ET}}$, instead of the onset of tillering and stem elongation, respectively.
\end{abstract}

Key words: Wheat-nitrogen fertilization. Seeding. Herbicides.

\footnotetext{
*Autor para correspondência

${ }^{1}$ Recebido para publicação em 31/01/2011; aprovado em 24/02/2012

Pesquisa financiada pela Universidade Federal de Santa Maria/UFSM e CAPES

${ }^{2}$ Programa de Pós-Graduação em Agronomia/Fitotecnia/CCR/UFSM, Santa Maria-RS, Brasil, alencarzanon@yahoo.com.br, a1000tontr@gmail.com ${ }_{3}^{3}$ Departamento de Fitotecnia/CCR/UFSM, Av. Roraima, Camobi, Santa Maria-RS, Brasil, 97.105-900, nstreck2@yahoo.com.br

${ }^{4}$ Programa de Pós-Graduação em Engenharia Agrícola/CCR/UFSM, Santa Maria-RS, Brasil, lidianewalter@gmail.com

${ }^{5}$ Universidade Federal do Pampa, Itaqui-RS, Brasil, cleberalb@yahoo.com.br
} 


\section{INTRODUÇÃO}

Entre os cereais de estação fria, o trigo (Triticum aestivum L.) é a espécie com maior área e produtividade a nível mundial, sendo cultivado entre latitudes de $60^{\circ} \mathrm{N}$ e $40^{\circ} \mathrm{S}$ e altitudes superiores a $3.000 \mathrm{~m}$ (MUNDSTOCK, 1999). Devido a esta ampla capacidade de adaptação e ao melhoramento genético, o trigo é a segunda cultura de grãos mais produzida no mundo (UNITED STATES DEPARTAMENT OF AGRICULTURE, 2011). A produtividade média de grãos de trigo no Brasil (1.813 $\left.\mathrm{kg} \mathrm{ha}^{-1}\right)$ situou-se abaixo da média mundial (2.718 $\mathrm{kg} \mathrm{ha}^{-1}$ ), no período de 1996 a 2006 (COMPANHIA NACIONAL DE ABASTECIMENTO, 2007). O Rio Grande do Sul é o segundo estado brasileiro em área de cultivo com 855.670 ha atrás do Paraná, com 1.308.792 ha (INSTITUTO BRASILEIRO DE GEOGRAFIA E ESTATÍSTICA, 2011).

Visando elevar a produtividade de trigo no Brasil é importante aumentar o conhecimento sobre a resposta de alguns fatores ambientais e práticas de manejo que afetam o desenvolvimento foliar e reprodutivo do trigo. É possível associar momentos específicos do desenvolvimento reprodutivo, como os estágios de duplo anel (DA) e espigueta terminal (ET), que são importantes na definição da produtividade final da cultura do trigo, com o desenvolvimento foliar através do número de folhas acumuladas no colmo (ROSA et al., 2009; SINCLAIR; JAMIESON, 2006; STRECK et al., 2007). Tal associação é de interesse prático, pois a época de determinadas práticas de manejo na cultura do trigo estão associadas a estes dois estágios de desenvolvimento. Por exemplo, de acordo com as recomendações (REUNIÃO DA COMISSÃO BRASILEIRA DE PESQUISA DE TRIGO, 2008) o nitrogênio em cobertura deve ser aplicado no início do perfilhamento, que ocorre um pouco antes do momento em que o ápice vegetativo começa a transformar-se em reprodutivo, pela diferenciação das primeiras espiguetas, que é o DA (PORTER et al., 1987). O restante do nitrogênio deve ser aplicado no início da elongação do colmo, que ocorre logo após a ET, quando todas as espiguetas já estão diferenciadas (STRECK et al., 2003a).

Outra prática de manejo que está diretamente associada com o período entre o DA e a ET é aplicação de herbicidas hormonais, que, segundo Rodrigues et al. (2006), é o período de menor sensibilidade do trigo a esses herbicidas. Porém, a determinação da data de ocorrência do DA e da ET é feita de maneira destrutiva (PORTER et al., 1987), o que é uma desvantagem pois necessita-se equipamento, pessoal treinado e demanda de tempo.

Portanto, o número de folhas é um indicador morfológico adicional e mais preciso do DA e da ET do que o início do perfilhamento e o início da elongação do colmo, e futuramente pode ser uma alternativa para melhorar a precisão nas práticas de manejo em trigo. Uma maneira de se representar o número de folhas é através da Escala de Haun (HAUN, 1973). Nessa escala, é medido o Estágio de Haun (HS), o qual representa o número de folhas expandidas mais a fração decimal do comprimento da lâmina da última folha (em expansão) em relação à penúltima folha.

Com ointuito de melhorar a precisão na determinação do início e fim da diferenciação floral em trigo a campo, conduziu-se esse trabalho com o objetivo de determinar o número de folhas, representado pelo HS, no DA e na ET de cultivares de trigo com diferentes ciclos de desenvolvimento e cultivados em diferentes datas de semeadura.

\section{MATERIAL E MÉTODOS}

O experimento de campo foi conduzido na área do Departamento de Fitotecnia, da Universidade Federal de Santa Maria (UFSM), em Santa Maria, RS (latitude $29^{\circ} 43^{\prime} \mathrm{S}$; longitude $53^{\circ} 43^{\prime} \mathrm{W}$; altitude $95 \mathrm{~m}$ ) durante três anos (2005, 2006 e 2007). O clima do local, segundo a classificação de Köppen, é do tipo Cfa, subtropical úmido com verões quentes e sem estação seca definida (KUINCHTNER; BURIOL, 2001). O solo do local é uma transição entre a Unidade de Mapeamento São Pedro (Argissolo Vermelho distrófico arênico) e a Unidade de Mapeamento Santa Maria (Alissolo Hipocrômico argilúvico típico).

Durante os três anos, foram realizadas semeaduras em intervalos de aproximadamente 45 dias, totalizando 13 datas de semeadura: 24/06/2005, 04/08/2005, 07/09/2005, $26 / 10 / 2005, \quad 21 / 12 / 2005, \quad 06 / 02 / 2006, \quad 21 / 03 / 2006$, 12/05/2006, 07/07/2006, 04/09/2006, 25/10/2006, 18/04/2007, 07/06/2007. Estas datas de semeadura se situam antes, durante e após o período recomendado para a semeadura do trigo na região de Santa Maria, que é de 11/05 a 20/06 (REUNIÃO DA COMISSÃO BRASILEIRA DE PESQUISA DE TRIGO, 2008). Os genótipos de trigo utilizados foram: CEP 51, CEP 52 e Nova Era, da Fundação Centro de Experimentação e Pesquisa (FUNDACEP) e BRS Louro, BRS 177 e BRS Tarumã, da Empresa Brasileira de Pesquisa Agropecuária (EMBRAPA). Esses genótipos são recomendados para o Rio Grande do Sul e foram selecionados por serem de empresas diferentes e por apresentarem ciclo de desenvolvimento distinto, variando de precoce (CEP 51 e BRS Louro), médio (CEP 52 e BRS 177) a tardio (Nova Era e BRS Tarumã) (REUNIÃO DA COMISSÃO BRASILEIRA DE PESQUISA DE TRIGO, 2006). O genótipo BRS Tarumã ainda é classificado como de duplo propósito (WENDT; DUCA; CAETANO, 2006), isto é, serve para a produção de forragem e de grãos.

A área experimental foi preparada de forma convencional. Na adubação de base aplicou-se a 
formulação 5-20-20 na dose de $300 \mathrm{~kg} \mathrm{ha}^{-1}$, no sulco de semeadura, de acordo com a análise química do solo, seguindo as Recomendações de Adubação e Calagem para os Estados do Rio Grande do Sul e Santa Catarina (COMISSÃO DE QUÍMICA E FERTILIDADE DO SOLO, 2004). Foi realizada adubação nitrogenada em cobertura na quantidade de $80 \mathrm{~kg} \mathrm{ha}^{-1}$ de nitrogênio parcelada em duas épocas, a primeira no DA e a segunda na ET.

Foi utilizado o delineamento em blocos completos casualizados, com quatro repetições em esquema fatorial com dois fatores: datas de semeadura e cultivares. A unidade experimental foi uma parcela formada de quatro linhas de plantas espaçadas de $0,20 \mathrm{~m}$ e de $2,40 \mathrm{~m}$ de comprimento e área de $1,92 \mathrm{~m}^{2}$. A densidade de semeadura foi de 330 sementes $\mathrm{m}^{-2}$. Considerou-se as duas linhas externas da parcela como bordadura e as duas linhas centrais como área útil da parcela $\left(0,96 \mathrm{~m}^{2}\right)$. Os tratos culturais, como controle de insetos, doenças e plantas invasoras, foram realizados sempre que necessários, de forma a evitar estresse por estes fatores bióticos.

A emergência (EM) foi considerada quando $50 \%$ das plantas em $0,5 \mathrm{~m}$ de cada fileira da parcela estavam acima do nível do solo. A contagem do número de plantas foi feita diariamente até obter-se número constante de plantas emergidas. Uma semana após a emergência, seis plantas de trigo em cada parcela foram selecionadas ao acaso e marcadas com arame colorido. Em todas as datas de semeadura, a partir do momento em que as plantas tinham três folhas visíveis no cartucho, coletaram-se, diariamente, duas plantas por parcela, as quais foram dessecadas e, com o auxílio de lupa, visualizado o estágio de DA e ET segundo Porter et al. (1987). A data do DA e da ET foi considerada quando $50 \%$ das plantas amostradas estavam nestes estágios. Na data em que ocorreram o DA e a ET, foram determinados, nas plantas marcadas da parcela, o número de folhas totalmente expandidas mais o comprimento da lâmina da penúltima e da última folha, para calcular o Estágio de Haun (HS) utilizando-se a fórmula (Equação 1):

$\mathrm{HS}=(\mathrm{NF}-1)+\mathrm{L}_{\mathrm{n}} / \mathrm{L}_{\mathrm{n}-1}$

em que NF é o número de folhas, $\mathrm{L}_{\mathrm{n}}$ é o comprimento da última folha $(\mathrm{cm})$ e $\mathrm{L}_{\mathrm{n}-1}$ é o comprimento da penúltima folha (cm) (McMASTER, 2005).

Quando o colar da folha bandeira estava visível foi realizada a contagem do número final de folhas (NFF) na haste principal das plantas marcadas. $\mathrm{O}$ dia do início do perfilhamento (primeiro perfilho visível) foi determinado nas plantas marcadas em apenas quatro datas de semeadura (07/09/2005, 26/10/2005, 21/12/2005 e 06/02/2006). Neste dia foi determinado também o HS.

As variáveis de interesse foram o Estágio de Haun no início do perfilhamento ( $\mathrm{HS}_{\mathrm{IP}}$ ), Estágio de Haun no Duplo Anel $\left(\mathrm{HS}_{\mathrm{DA}}\right)$, Estágio de Haun na Espigueta
Terminal $\left(\mathrm{HS}_{\mathrm{ET}}\right)$, o número de folhas entre o DA e a ET, representada pela diferença do $\mathrm{HS}_{\mathrm{ET}}$ menos $\mathrm{HS}_{\mathrm{DA}}\left[\mathrm{HS}_{\mathrm{ET}}{ }^{-}\right.$ $\mathrm{HS}_{\mathrm{DA}}$ ], e o número de folhas que, na $\mathrm{ET}$, restam a aparecer até a folha bandeira representado pela diferença entre NFF e o $\mathrm{HS}_{\mathrm{ET}}\left[\mathrm{NFF}-\mathrm{HS}_{\mathrm{ET}}\right]$. A análise estatística foi realizada considerando um experimento bi-fatorial, onde o fator A foi cultivar (seis níveis) e o fator D foi data de semeadura (13 níveis). As médias dessas variáveis foram distinguidas pelo teste de Tukey $(\mathrm{p}<0,05)$. Com o objetivo de verificar se as variáveis dependentes tem relação com o NFF (STRECK et al., 2009), foi realizada também uma regressão linear simples $(\mathrm{y}=\mathrm{a}+\mathrm{bx}+$ erro experimental) entre as variáveis $\mathrm{HS}_{\mathrm{DA}}, \mathrm{HS}_{\mathrm{ET}},\left[\mathrm{HS} \mathrm{ET}_{-}-\mathrm{HS}_{\mathrm{DA}}\right],\left[\mathrm{NFF}-\mathrm{HS}_{\mathrm{ET}}\right]$ (variável dependente) e o NFF (variável independente). A qualidade do ajuste do modelo foi com base na significância do teste F para a hipótese H0 (o coeficiente angular não é diferente de zero) x a hipótese H1 (o coeficiente angular é diferente de zero). Todas as análises estatísticas foram realizadas com auxílio do pacote estatístico SAS e significância de $5 \%$ de probabilidade do erro tipo I.

\section{RESULTADOS E DISCUSSÃO}

Durante a coleta da variável $\mathrm{HS}_{\mathrm{IP}}$ foi observado que, em algumas plantas em determinadas datas de semeadura, o primeiro perfilho não correspondia ao $\mathrm{T} 1$ (perfilho de axila da primeira folha) e sim ao T2 (perfilho de axila da segunda folha) e às vezes até o T3 (perfilho de axila da terceira folha). Como o aparecimento de cada perfilho é sincronizado no tempo e o HS é diferente no aparecimento de cada perfilho (RITCHIE, 1991), fez-se duas análises do $\mathrm{HS}_{\mathrm{IP}}$, uma considerando todas as plantas independente do perfilho que apareceu $\left(\mathrm{HS}_{\mathrm{IP}}\right)$ e outra considerando-se apenas o aparecimento do perfilho T1 ( $\left.\mathrm{HS}_{\mathrm{IP}(\mathrm{Tl})}\right)$ (Tabela 3).

A análise de variância mostrou efeito significativo dos fatores cultivar e data de semeadura para todas as variáveis (Tabela 1). O quadrado médio foi maior para o fator cultivar do que para o fator data de semeadura, exceto para as variáveis $\mathrm{HS}_{\mathrm{IP}}$ e $\left(\mathrm{HS}_{\mathrm{ET}}-\mathrm{HS}_{\mathrm{DA}}\right)$, o que indica que a maioria das variáveis foram mais afetadas pela constituição genética do que pelo efeito ambiental, exceto o $\mathrm{HS}_{\mathrm{IP}}$ e o $\left(\mathrm{HS}_{\mathrm{ET}}-\mathrm{HS}_{\mathrm{DA}}\right)$, as quais foram mais afetadas pelo ambiente em que as plantas completaram o seu ciclo (Tabela 1). $\mathrm{O}$ coeficiente de variação variou de 3,57 a 13,79. De acordo com Gomes (1990), a precisão experimental neste estudo pode ser classificada como alta, pois os coeficientes de variação para a maioria das variáveis analisadas foram inferiores ou próximos a $10 \%$, ou seja, devido ao CV ser baixo, menor foi o erro experimental e por consequência maior a precisão ou qualidade deste ensaio.

As análises de variância (Tabela 1) mostraram efeitos significativos da interação cultivar $\mathrm{x}$ data de 
Tabela 1 - Valores do quadrado médio da ANOVA para as variáveis número final de folhas no colmo principal (NFF), Estágio de Haun no início do perfilhamento $\left(\mathrm{HS}_{\mathrm{IP}}\right)$, Estágio de Haun no início do perfilhamento considerando-se apenas o aparecimento do perfilho T1 $\left(\mathrm{HS}_{\mathrm{IPT} 1}\right)$, Estágio de Haun no duplo anel $\left(\mathrm{HS}_{\mathrm{DA}}\right)$ e na espigueta terminal $\left(\mathrm{HS}_{\mathrm{ET}}\right)$, diferença entre $\mathrm{HS}_{\mathrm{ET}}$ e o $\mathrm{HS}_{\mathrm{DA}}$ e diferença entre $\mathrm{NFF}$ o $\mathrm{HS}_{\mathrm{ET}}$ para seis cultivares de trigo em treze datas de semeadura em cultivo em Santa Maria, RS, Brasil, entre 2005 e 2007. Santa Maria, RS, 2011

\begin{tabular}{|c|c|c|c|c|c|c|c|}
\hline \multirow{3}{*}{ Causas de variação } & \multicolumn{7}{|c|}{ Quadrado Médio } \\
\hline & $\mathrm{NFF}$ & $\mathrm{HS}_{\mathrm{IP}}$ & $\mathrm{HS}_{\mathrm{IP}}(\mathrm{T} 1)$ & $\mathrm{HS}_{\mathrm{DA}}$ & $\mathrm{HS}_{\mathrm{ET}}$ & $\mathrm{HS}_{\mathrm{ET}}-\mathrm{HS}_{\mathrm{DA}}$ & $\mathrm{NFF}-\mathrm{HS}_{\mathrm{ET}}$ \\
\hline & \multicolumn{7}{|c|}{$2005-2007$} \\
\hline Bloco & $0,12 *$ & $0,08 *$ & $0,11 *$ & $0,08 *$ & $0,06^{*}$ & $0,04 *$ & $0,06^{*}$ \\
\hline Cultivar (A) & $150,22 *$ & $0,96 *$ & $0,92 *$ & $142,4^{*}$ & $115,76^{*}$ & $0,61 *$ & $6,54 *$ \\
\hline Data de Semeadura (D) & $31,9 *$ & $12,82 *$ & $12,22 *$ & $17,85^{*}$ & $26,71 *$ & $4,58 *$ & $3,91 *$ \\
\hline$A \times D$ & $1,57 *$ & $0,12 *$ & $0,17 *$ & $2,37 *$ & $1,9 *$ & $0,78 *$ & $1,06^{*}$ \\
\hline Coeficiente de Variação (\%) & 4,16 & 6,14 & 7,90 & 4,60 & 3,57 & 12,35 & 13,79 \\
\hline
\end{tabular}

* Significativo a $5 \%$

semeadura para as variáveis NFF (Tabela 2), $\mathrm{HS}_{\mathrm{IP}}$ e $\mathrm{HS}_{\mathrm{IP}(\mathrm{T} 1)}$ (Tabela 3), $\mathrm{HS}_{\mathrm{DA}}$ (Tabela 4), $\mathrm{HS}_{\mathrm{ET}}$ (Tabela 5), $\mathrm{HS}_{\mathrm{ET}}-\mathrm{HS}_{\mathrm{DA}}$

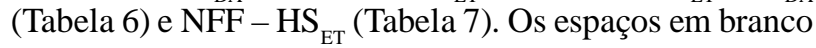
nas Tabelas 2 a 7 representam as datas de semeadura, em que as plantas não completaram as variáveis medidas neste trabalho. Por exemplo, as plantas da cultivar BRS Tarumã quando semeada nas datas 04/09/2006 e 25/10/2006 não atingiram o DA e a ET por não terem sido expostas a temperaturas vernalizantes e assim permaneceram vegetativas (Tabela 5 e 6).

Em algumas situações não foi possível fazer a determinação do DA e/ou ET devido ao rápido desenvolvimento e curta duração das fases, o que acarretou na perda de algumas repetições, como aconteceu com as cultivares CEP 51, Nova Era e BRS Tarumã na data de semeadura de 07/06/2007 (Tabela 2), com as cultivares BRS 177, Nova Era, BRS Tarumã na data de semeadura de 24/06/2005 e com as cultivares BRS Louro, CEP 52, BRS 177, BRS Tarumã na data de semeadura de 07/06/2007 (Tabela 3). A perda dessas repetições acarretou em tratamentos com uma única repetição, logo não foi possível realizar a análise estatística na interação cultivar dentro das datas de semeadura e por isso não há letra minúscula ligando cultivares nas datas de semeadura 04/09/2006 e 25/10/2006 nas Tabelas 3; 5; 6 e 7.

O NFF no colmo principal variou com a cultivar e a data de semeadura, de 8,5 folhas na cultivar BRS Louro semeada em 25/10/2006 até 20,2 folhas na cultivar BRS Tarumã semeada em 06/02/2006 (Tabela 2). Entre as cultivares, o maior NFF médio foi na cultivar tardia BRS Tarumã, menor nas duas cultivares de ciclo precoce (CEP 51 e BRS Louro) e intermediário nas outras cultivares (CEP 52 e BRS 177). Quando não houve dados de NFF para a cultivar BRS Tarumã (datas 21/12/2005, 07/07/2006 e 25/10/2006), o maior NFF foi da cultivar Nova Era, a qual geralmente diferiu apenas de uma ou no máximo das duas cultivares mais precoces. Entre as datas de semeadura, aquelas realizadas durante os meses mais quentes tiveram maior NFF, especialmente na cultivar BRS Tarumã, e os menores valores de NFF foram nas semeaduras realizadas na primavera. Esses resultados confirmam resultados anteriores que em poáceas o NFF é diretamente proporcional à duração do ciclo total de desenvolvimento e do grupo de maturação das cultivares (STRECK et al., 2006; STRECK et al., 2009; WALTER et al., 2009).

A cultivar BRS Tarumã apresentou um elevado NFF, característica das cultivares de duplo propósito, que podem ser pastejadas no inverno, e ainda produzem área foliar suficiente para sustentar o enchimento dos grãos na primavera. De acordo com Alberto et al. (2009a), a cultivar BRS Louro não é sensível à vernalização, e assim quando exposta a temperatura e fotoperíodo elevados, como acontecem nas semeaduras durante a primavera e o ciclo acontece durante o verão, esta cultivar apresentou os menores valores de NFF.

Já a cultivar BRS Tarumã é muito sensível à vernalização (ALBERTO et al., 2009a, 2009b), e, portanto apresentou uma resposta típica de trigo de inverno (STRECK et al., 2003a,2003b), com os maiores valores do NFF nas semeaduras de primavera e verão quando as plantas não foram expostas a temperaturas vernalizantes durante a estação de crescimento. As plantas de trigo apresentaram valores intermediários de NFF nas semeaduras de outono, quando o seu ciclo de desenvolvimento ocorreu durante o período recomendado para a cultura. Essa grande variação no NFF devido aos diferentes genótipos e as diferentes datas de semeadura fornece um amplo e apropriado conjunto de dados para o teste da hipótese deste estudo, que foi de que o HS pode ser usado como indicador morfológico das plantas para 
Tabela 2 - Número final de folhas (NFF) no colmo principal em seis cultivares de trigo em treze datas de semeadura em cultivo em Santa Maria, RS, Brasil, entre 2005 e 2007. Santa Maria, RS, 2011

\begin{tabular}{lccclcc}
\hline \multirow{2}{*}{ Datas de semeadura } & \multicolumn{7}{c}{ Cultivares } \\
\cline { 2 - 7 } & BRS Louro & CEP 52 & BRS 177 & CEP 51 & Nova Era & BRS Tarumã \\
\hline $24 / 6 / 2005$ & $10,1 \mathrm{BCDc} *$ & $10,4 \mathrm{Efc}$ & $10,2 \mathrm{CDEc}$ & $11,6 \mathrm{CDEb}$ & $11,4 \mathrm{Efb}$ & $16,4 \mathrm{BCa}$ \\
$4 / 8 / 2005$ & $9,6 \mathrm{CDc}$ & $9,7 \mathrm{Fc}$ & $9,9 \mathrm{DEc}$ & $10,8 \mathrm{DEFGb}$ & $10,8 \mathrm{Fb}$ & $15,1 \mathrm{Ca}$ \\
$7 / 9 / 2005$ & $9,5 \mathrm{CDd}$ & $10,1 \mathrm{EFbcd}$ & $9,6 \mathrm{Ecd}$ & $10,5 \mathrm{EFGbc}$ & $10,6 \mathrm{Fb}$ & $16,0 \mathrm{Ca}$ \\
$26 / 10 / 2005$ & $8,8 \mathrm{Dc}$ & $9,6 \mathrm{Fc}$ & $9,5 \mathrm{Ec}$ & $9,9 \mathrm{Gbc}$ & $11,2 \mathrm{EFb}$ & $15,0 \mathrm{Ca}$ \\
$21 / 12 / 2005+$ & $10,8 \mathrm{ABCb}$ & $12,7 \mathrm{Bab}$ & $11,4 \mathrm{Bab}$ & $12,3 \mathrm{BCab}$ & $14,0 \mathrm{Ba}$ & -+ \\
$6 / 2 / 2006$ & $12,0 \mathrm{Ad}$ & $15,3 \mathrm{Ab}$ & $13,3 \mathrm{Acd}$ & $14,0 \mathrm{Abc}$ & $15,8 \mathrm{Ab}$ & $20,2 \mathrm{Aa}$ \\
$21 / 3 / 2006$ & $10,7 \mathrm{ABCd}$ & $12,3 \mathrm{BCbc}$ & $10,9 \mathrm{BCd}$ & $11,7 \mathrm{CDcd}$ & $13,0 \mathrm{BCDb}$ & $17,7 \mathrm{Ba}$ \\
$12 / 5 / 2006$ & $9,8 \mathrm{BCDc}$ & $9,8 \mathrm{Fc}$ & $9,8 \mathrm{Ec}$ & $10,5 \mathrm{EFGbc}$ & $11,0 \mathrm{Fb}$ & $18,6 \mathrm{Ca}$ \\
$7 / 7 / 2006+$ & $9,3 \mathrm{CDa}$ & $10,5 \mathrm{EFb}$ & $11,1 \mathrm{Ba}$ & $11,2 \mathrm{CDEFa}$ & $11,0 \mathrm{Fab}$ & -+ \\
$4 / 9 / 2006$ & $9,7 \mathrm{BCDc}$ & $9,9 \mathrm{Fc}$ & $9,4 \mathrm{Ec}$ & $10,1 \mathrm{FGc}$ & $11,6 \mathrm{DEFb}$ & $15,5 \mathrm{Ca}$ \\
$25 / 10 / 2006+$ & $8,5 \mathrm{Dc}$ & $11,0 \mathrm{DEab}$ & $9,7 \mathrm{Ebc}$ & $11,0 \mathrm{DEFGab}$ & $11,9 \mathrm{CDEFa}$ & -+ \\
$18 / 4 / 2007$ & $11,3 \mathrm{ABc}$ & $11,6 \mathrm{CDbc}$ & $10,7 \mathrm{BCDc}$ & $12,3 \mathrm{BCb}$ & $12,5 \mathrm{CDEb}$ & $15,2 \mathrm{Ca}$ \\
$7 / 6 / 2007$ & $12,3 \mathrm{Ac}$ & $12,3 \mathrm{BCc}$ & $11,2 \mathrm{Bd}$ & $13,0 \mathrm{ABb}$ & $13,1 \mathrm{BCb}$ & $14,8 \mathrm{Ca}$ \\
\hline
\end{tabular}

* Médias seguidas por mesma letra maiúscula nas colunas e minúscula nas linhas não diferem entre si pelo Teste Tukey a 5\% de erro. +Plantas não atingiram o número final de folhas nestas datas de semeadura

identificar o DA e a ET em trigo, e que o $\mathrm{HS}_{\mathrm{DA}}$ e o $\mathrm{HS}_{\mathrm{ET}}$ podem variar entre genótipos e datas de semeadura.

$\mathrm{O} \mathrm{HS}_{\mathrm{IP}}$ variou entre cultivares de 2,95 a 5,10, sendo maior na BRS Louro (cultivar precoce) com exceção apenas da data de semeadura de 06/02/2006 quando não houve diferenças entre as cultivares (Tabela 3). As outras cultivares não diferiram entre si exceto na data de semeadura de 07/09/2005, quando a BRS 177 diferiu da CEP 51. Quando considerado apenas as plantas que emitiram o T1 como primeiro perfilho, o HS ${ }_{\mathrm{IP}(\mathrm{T})}$ foi menor em relação às plantas que emitiram o T2 ou T3 como primeiro perfilho, e neste caso apenas a BRS Louro foi diferente das demais nas datas de 07/09/2005 e 26/10/2005. Entre as datas de semeadura, o $\mathrm{HS}_{\mathrm{IP}}$ e $\mathrm{HS}_{\mathrm{IP}(\mathrm{TI})}$ foram maiores nas semeaduras no verão $(21 / 12 / 2005$ e 06/02/2006) do que nas da primavera (07/09/2005 e 26/10/2005). Estes resultados indicam que, em termos de número de folhas, o perfilhamento ocorre mais tarde na cultivar precoce (BRS Louro) e em semeaduras nos meses mais quentes.

Com relação ao $\mathrm{HS}_{\mathrm{DA}}$ entre as cultivares, esta variável sempre foi maior na BRS Tarumã, cultivar mais tardia, onde variou de 9,82 a 18,13 folhas, e menor nas duas cultivares mais precoces (BRS Louro e CEP 52), onde variou de 4,20 a 7,28 folhas (Tabela 4). Nas datas de semeadura em que não há dados da cultivar BRS Tarumã, $o$ maior $\mathrm{HS}_{\mathrm{DA}}$ médio foi na cultivar Nova Era (7,27 folhas), a qual também é classificada como menos tardia que a BRS
Tarumã (WALTER et al., 2009). As outras duas cultivares (CEP 51 e BRS 177) tiveram $\mathrm{HS}_{\mathrm{DA}}$ intermediário entre as cultivares tardias e precoces, intercalando a sua posição em função da data de semeadura, ora diferindo apenas da cultivar mais tardia (26/10/2005), ora diferindo das duas cultivares mais tardias (06/02/2006, 21/03/2006, 12/05/2006), mas não diferindo das cultivares precoces.

Entre as datas de semeadura, aquelas realizadas nos meses mais quentes (dezembro a março) tiveram os maiores valores de $\mathrm{HS}_{\mathrm{DA}}$, especialmente para as duascultivarestardias, NovaEra e BRS Tarumã, que tiveramum $\mathrm{HS}_{\mathrm{DA}}$ médio de 8,26 e 15,34 folhas, respectivamente. Estes resultados indicam relação entre o $\mathrm{HS}_{\mathrm{DA}}$ e o ciclo de desenvolvimento, o qual tem relação com o NFF (WALTER et al., 2009). A análise da relação entre $\mathrm{HS}_{\mathrm{DA}}$ e NFF considerando o conjunto dos dados de todas as cultivares e datas de semeadura confirmam esta inferência de tendência geral pois houve uma relação linear significativa (F calculado de 442,66 para a hipótese H0: o coeficiente angular não é diferente de zero x hipótese H1: o coeficiente angular é diferente de zero) $c o m R^{2}=0,74$ e aumento médio de $\mathrm{HS}_{\mathrm{DA}}$ de 0,79 folhas para cada aumento unitário de NFF (Figura 1a).

Para o $\mathrm{HS}_{\mathrm{ET}}$, os resultados seguiram a mesma tendência do $\mathrm{HS}_{\mathrm{DA}}$ (Tabela 5). A cultivar mais tardia (BRS Tarumã) apresentou um $\mathrm{HS}_{\mathrm{ET}}$ médio de 14,03 folhas, maior do que $\mathrm{HS}_{\mathrm{ET}}$ médio das outras cultivares, que variou de 7,7 (cultivares BRS Louro e CEP 52) a 8,9 folhas (cultivar 
Tabela 3 - Estágio de Haun no início do perfilhamento considerando qualquer perfilho $\left(\mathrm{HS}_{\mathrm{IP}}\right)$ e considerando apenas o perfilho $\mathrm{T} 1\left(\mathrm{HS}_{\mathrm{IP}(\mathrm{T} 1}\right)$ no colmo principal em seis cultivares de trigo e quatro datas de semeadura em cultivo em Santa Maria, RS, Brasil, entre 2005 e 2007. Santa Maria, RS, 2011

\begin{tabular}{lcccc}
\hline \multirow{2}{*}{ Cultivares } & \multicolumn{4}{c}{ Datas de semeadura } \\
\cline { 2 - 5 } & $7 / 9 / 2005$ & $26 / 10 / 2005$ & $21 / 12 / 2005$ & $6 / 2 / 2006$ \\
\cline { 2 - 5 } & $4,10 \mathrm{Ba}^{*}$ & $3,74 \mathrm{Ba}$ & $4,40 \mathrm{ABa}$ & $5,10 \mathrm{Aa}$ \\
\hline BRS Louro & $3,18 \mathrm{Cbc}$ & $3,19 \mathrm{Cb}$ & $3,77 \mathrm{Bab}$ & $4,87 \mathrm{Aa}$ \\
CEP 52 & $2,95 \mathrm{Cc}$ & $3,17 \mathrm{Cb}$ & $3,95 \mathrm{Bab}$ & $4,53 \mathrm{Aa}$ \\
BRS 177 & $3,31 \mathrm{Bb}$ & $3,25 \mathrm{Bb}$ & $3,74 \mathrm{Bb}$ & $4,96 \mathrm{Aa}$ \\
CEP 51 & $3,18 \mathrm{Cbc}$ & $3,27 \mathrm{Cb}$ & $3,83 \mathrm{Bab}$ & $4,64 \mathrm{Aa}$ \\
Nova Era & $3,07 \mathrm{Cbc}$ & $3,11 \mathrm{Cb}$ & $4,12 \mathrm{Bab}$ & $4,89 \mathrm{Aa}$ \\
BRS Tarumã & & Considerando apenas T1 - HS & \\
\hline & $3,87 \mathrm{Ba}$ & $3,63 \mathrm{Ba}$ & $4,42 \mathrm{ABa}$ & $5,10 \mathrm{Aa}$ \\
\hline BRS Louro & $3,18 \mathrm{Bb}$ & $3,19 \mathrm{Bb}$ & $3,60 \mathrm{Ba}$ & $4,89 \mathrm{Aa}$ \\
CEP 52 & $2,95 \mathrm{Bb}$ & $3,12 \mathrm{Bb}$ & $4,02 \mathrm{Aa}$ & $4,47 \mathrm{Aa}$ \\
BRS 177 & $3,31 \mathrm{Bb}$ & $3,25 \mathrm{Bb}$ & $3,67 \mathrm{Ba}$ & $5,08 \mathrm{Aa}$ \\
CEP 51 & $3,17 \mathrm{Bb}$ & $3,27 \mathrm{Bb}$ & $3,67 \mathrm{Ba}$ & $4,43 \mathrm{Aa}$ \\
Nova Era & $3,07 \mathrm{Cb}$ & $3,11 \mathrm{Cb}$ & $4,17 \mathrm{Ba}$ & $4,96 \mathrm{Aa}$ \\
BRS Tarumã & & &
\end{tabular}

* Médias seguidas por mesma letra maiúscula nas linhas e minúscula nas colunas não diferem entre si pelo Teste Tukey a $5 \%$ de erro

Tabela 4 - Estágio de Haun no duplo anel $\left(\mathrm{HS}_{\mathrm{DA}}\right)$ no colmo principal em seis cultivares de trigo e dez datas de semeadura em cultivo em Santa Maria, RS, Brasil, entre 2005 e 2007. Santa Maria, RS, 2011

\begin{tabular}{llllllc}
\hline \multirow{2}{*}{ Datas de semeadura } & \multicolumn{5}{c}{ Cultivares } \\
\cline { 2 - 7 } & BRS Louro & CEP 52 & BRS 177 & CEP 51 & Nova Era & BRS Tarumã \\
\hline $26 / 10 / 2005$ & $5,65 \mathrm{BCDb}^{*}$ & $5,32 \mathrm{Db}$ & $5,51 \mathrm{BCb}$ & $5,65 \mathrm{Deb}$ & $5,64 \mathrm{Fb}$ & $12,73 \mathrm{Ba}$ \\
$21 / 12 / 2005+$ & $6,34 \mathrm{ABb}$ & $7,12 \mathrm{ABb}$ & $6,61 \mathrm{Bb}$ & $8,26 \mathrm{Aa}$ & $8,61 \mathrm{Aa}$ & $-^{+}$ \\
$6 / 2 / 2006$ & $7,13 \mathrm{Ac}$ & $7,28 \mathrm{Ac}$ & $8,78 \mathrm{Ab}$ & $7,26 \mathrm{BCc}$ & $9,02 \mathrm{Ab}$ & $18,13 \mathrm{Aa}$ \\
$21 / 3 / 2006$ & $6,21 \mathrm{BCc}$ & $6,00 \mathrm{DCc}$ & $6,28 \mathrm{Bc}$ & $5,88 \mathrm{DEc}$ & $7,14 \mathrm{BCDb}$ & $12,54 \mathrm{Ba}$ \\
$12 / 5 / 2006$ & $5,60 \mathrm{BCDc}$ & $5,25 \mathrm{Dc}$ & $5,58 \mathrm{BCc}$ & $5,18 \mathrm{Ec}$ & $6,25 \mathrm{DEFb}$ & $9,82 \mathrm{Ca}$ \\
$7 / 7 / 2006+$ & $5,48 \mathrm{BCDb}$ & $5,85 \mathrm{DCb}$ & $6,10 \mathrm{Bab}$ & $6,55 \mathrm{CDa}$ & $6,14 \mathrm{EFab}$ & $-^{+}$ \\
$4 / 9 / 2006+\dagger$ & $5,45 \mathrm{CD} \dagger$ & $5,35 \mathrm{D} \dagger$ & $6,38 \mathrm{~B} \dagger$ & $6,43 \mathrm{CD} \dagger$ & $6,62 \mathrm{CDE} \dagger$ & $-{ }^{+}$ \\
$25 / 10 / 2006+\dagger$ & $4,2 \mathrm{E} \dagger$ & $5,11 \mathrm{D} \dagger$ & $4,59 \mathrm{C} \dagger$ & $6,55 \mathrm{CD} \dagger$ & $7,69 \mathrm{~B} \dagger$ & $-{ }^{+}$ \\
$18 / 4 / 2007$ & $6,11 \mathrm{BCc}$ & $6,32 \mathrm{BCc}$ & $5,86 \mathrm{BCc}$ & $7,58 \mathrm{ABb}$ & $7,40 \mathrm{BCb}$ & $9,86 \mathrm{Ca}$ \\
$7 / 6 / 2007 \#$ & $5,18 \mathrm{Db}$ & $5,93 \mathrm{DCa}$ & $5,40 \mathrm{BCb}$ & $-\#$ & $-\#$ & $-\#$ \\
\hline
\end{tabular}

* Médias seguidas por mesma letra maiúscula nas colunas e minúscula nas linhas não diferem entre si pelo Teste Tukey a 5\% de erro. ${ }^{+}$Plantas não atingiram o estágio de duplo anel nas datas de semeadura. 'Não foi realizada a análise estatística na interação cultivar dentro das datas de semeadura

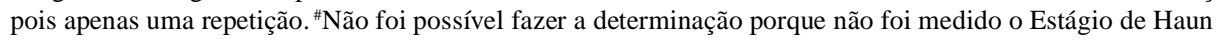

Nova Era). As semeaduras realizadas nos meses mais frios (abril a novembro) tiveram um $\mathrm{HS}_{\mathrm{DA}}$ médio de 8,09 folhas, enquanto que as semeaduras realizadas nos meses mais quentes tiveram um $\mathrm{HS}_{\mathrm{DA}}$ médio de 10,20 folhas. Ou seja, esses resultados também indicam uma relação entre $\mathrm{HS}_{\mathrm{ET}}$ com o ciclo de desenvolvimento (que é função do grupo de maturação da cultivar e da data de semeadura), o que se confirma na Figura $1 b$ em que a relação linear entre o 
Figura 1 - Relação entre (a,b) o Estágio de Haun no duplo anel $\left(\mathrm{HS}_{\mathrm{DA}}\right)$ e na espigueta terminal $\left(\mathrm{HS}_{\mathrm{ET}}\right)$ e o número final de folhas (NFF) e entre (c,d) a diferença entre o Estágio de Haun no DA e na ET e a diferença entre o NFF e o Estágio de Haun na espigueta terminal e número final de folhas (NFF) no colmo principal de seis cultivares de trigo (BRS Louro, CEP 51, BRS 177 CEP 52, Nova Era e BRS Tarumã) em treze datas de semeadura em cultivo em Santa Maria, RS, Brasil, entre 2005 e 2007. Cada ponto é uma repetição (média de seis plantas). Santa Maria, RS, 2011
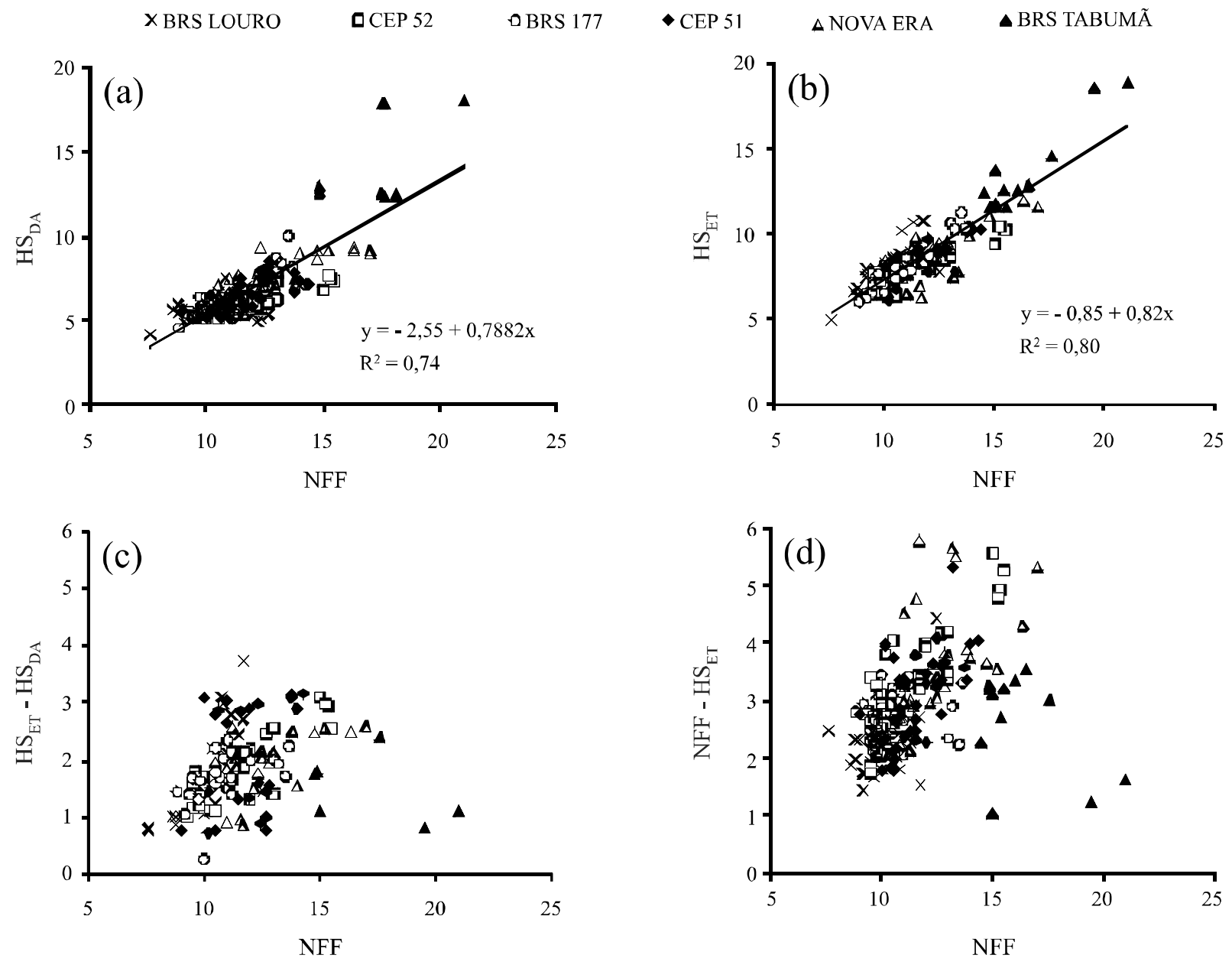

$\mathrm{HS}_{\mathrm{ET}}$ e o NFF foi significativa ( $\mathrm{F}$ calculado de 759,74 para a hipótese H0: o coeficiente angular não é diferente de zero x hipótese $\mathrm{H} 1$ : o coeficiente angular é diferente de zero) com $\mathrm{R}^{2}=0,80$, considerando-se o conjunto dos dados de todas as cultivares e datas de semeadura e aumento médio de 0,82 folhas no $\mathrm{HS}_{\mathrm{ET}}$ para cada aumento unitário de NFF.

Já para as variáveis $\left[\mathrm{HS}_{\mathrm{ET}}-\mathrm{HS}_{\mathrm{DA}}\right]$ e $\left[\mathrm{NFF}-\mathrm{HS}_{\mathrm{ET}}\right]$, o efeito de cultivar e datas de semeadura foi significativo (Tabela 6), porém não houve uma tendência clara, nem relação com o NFF, pois a regressão linear não foi significativa e o $\mathrm{R}^{2}$ foi de 0,10 e 0,16 para as duas variáveis (Figuras 1c e 1d). Entre cultivares, a CEP 51 apresentou o maior ( 3,06 folhas) e o menor ( 0,73 folhas $)$ $\left[\mathrm{HS}_{\mathrm{ET}}-\mathrm{HS}_{\mathrm{DA}}\right]$ e entre datas de semeadura, aquelas realizadas nos meses mais quentes apresentaram um

$\left[\mathrm{HS}_{\mathrm{ET}}-\mathrm{HS}_{\mathrm{DA}}\right]$ médio de 2,02 folhas, enquanto que as outras apresentaram um $\left[\mathrm{HS}_{\mathrm{ET}}-\mathrm{HS}_{\mathrm{DA}}\right]$ médio de 1,52 folhas.

Com respeito à variável número de folhas que ainda faltam aparecer até a folha bandeira a partir da ET $\left[\mathrm{NFF}-\mathrm{HS}_{\mathrm{ET}}\right]$, as diferenças entre genótipos e datas de semeadura foram pequenas, mas significativas (Tabela 7). A cultivar CEP 52 foi a que apresentou maior [NFF - $\mathrm{HS}_{\mathrm{ET}}$ ] (5,14 folhas), e a cultivar BRS Tarumã apresentou o menor número de folhas que ainda faltam aparecer até ser atingido o NFF (1,08 folhas). Assim, de forma geral estes resultados sugerem que o número de folhas entre o DA e a ET pode variar entre 1 e 4 folhas e o número de folhas que ainda faltam para emergir a partir da ET pode variar entre 1 e 6 folhas, mas sem relação com o NFF.

Os resultados deste estudo indicam que oinício(DA) e o final (ET) da diferenciação dos primórdios florais em 
Tabela 5 - Estágio de Haun na espigueta terminal $\left(\mathrm{HS}_{\mathrm{ET}}\right)$ no colmo principal em seis cultivares de trigo e doze datas de semeadura em cultivo em Santa Maria, RS, Brasil, entre 2005 e 2007. Santa Maria, RS, 2011

\begin{tabular}{|c|c|c|c|c|c|c|}
\hline \multirow[t]{2}{*}{ Datas de semeadura } & \multicolumn{6}{|c|}{ Cultivares } \\
\hline & BRS Louro & CEP 52 & BRS 177 & CEP 51 & Nova Era & BRS Tarumã \\
\hline 24/6/2005\# & $8,20 \mathrm{BCb}^{*}$ & $8,04 \mathrm{BCb}$ & _\# & $9,21 \mathrm{Ba}$ & _\# & _\# \\
\hline 4/8/2005 & 7,68 CDb & 7,74 BCDb & $7,33 \mathrm{CDb}$ & $7,72 \mathrm{Db}$ & 7,74 Eb & $12,13 \mathrm{Ca}$ \\
\hline 7/9/2005+ & 7,73 CD & 7,31 CDEb & 7,38 CDbc & $8,59 \mathrm{BCa}$ & 8,41 CDEa & $-^{+}$ \\
\hline $26 / 10 / 2005$ & $6,62 \mathrm{~Eb}$ & $6,45 \mathrm{~Eb}$ & 6,64 Deb & $6,38 \mathrm{~Eb}$ & $6,56 \mathrm{Fb}$ & $14,45 \mathrm{Ba}$ \\
\hline $21 / 12 / 2005+$ & 7,68 CDc & $8,53 \mathrm{Bb}$ & $8,52 \mathrm{Bb}$ & $9,00 \mathrm{Bb}$ & $10,23 \mathrm{Ba}$ & ${ }_{-}^{+}$ \\
\hline $6 / 2 / 2006$ & $10,56 \mathrm{Ac}$ & $10,13 \mathrm{Ac}$ & $10,64 \mathrm{Abc}$ & $10,27 \mathrm{Ac}$ & $11,60 \mathrm{Ab}$ & 19,04 Aa \\
\hline $21 / 3 / 2006$ & $8,40 \mathrm{BCbc}$ & $8,35 \mathrm{Bbc}$ & 7,89 BCc & $8,74 \mathrm{Bbc}$ & $9,23 \mathrm{BCb}$ & $14,66 \mathrm{Ba}$ \\
\hline $12 / 5 / 2006$ & $7,18 \mathrm{DEc}$ & $6,87 \mathrm{DEc}$ & $7,12 \mathrm{CDc}$ & $8,00 \mathrm{CDb}$ & $8,13 \mathrm{DEb}$ & $12,26 \mathrm{Ca}$ \\
\hline $4 / 9 / 2006+\dagger$ & $6,83 \mathrm{E} \dagger$ & $6,53 \mathrm{E} \dagger$ & 7,66 BCD $\dagger$ & $7,85 \mathrm{D} \dagger$ & $9,19 \mathrm{BCD} \dagger$ & ${ }_{-}^{+}$ \\
\hline $25 / 10 / 2006+\dagger$ & $4,99 \mathrm{~F} \dagger$ & $6,39 \mathrm{E} \dagger$ & $6,03 \mathrm{E} \dagger$ & $7,87 \mathrm{D} \dagger$ & $9,83 \mathrm{~B} \dagger$ & $-_{+}^{+}$ \\
\hline $18 / 4 / 2007$ & $8,86 \mathrm{Bbc}$ & $8,36 \mathrm{Bcd}$ & $7,95 \mathrm{BCd}$ & $9,05 \mathrm{Bbc}$ & $9,35 \mathrm{BCb}$ & $11,62 \mathrm{Ca}$ \\
\hline 7/6/2007†\# & _\# & _\# & _\# & $7,86 \mathrm{D} \dagger$ & 7,67 E† & _\# \\
\hline
\end{tabular}

* Médias seguidas por mesma letra maiúscula nas colunas e minúscula nas linhas não diferem entre si pelo Teste Tukey a 5\% de erro. ${ }^{+}$Plantas não atingiram o estágio de espigueta terminal nas datas de semeadura. `Não foi realizada a análise estatística na interação cultivar dentro das datas de semeadura pois apenas uma repetição. "Não foi possível fazer a determinação porque não foi medido o Estágio de Haun

Tabela 6 - Diferença entre o Estágio de Haun na espigueta terminal e no duplo anel $\left(\mathrm{HS}_{\mathrm{ET}}-\mathrm{HS}_{\mathrm{DA}}\right)$ no colmo principal em seis cultivares de trigo e oito datas de semeadura em cultivo em Santa Maria, RS, Brasil, entre 2005 e 2007. Santa Maria, RS, 2011

\begin{tabular}{llllllc}
\hline \multirow{2}{*}{ Datas de semeadura } & \multicolumn{5}{c}{ Cultivares } \\
\cline { 2 - 7 } & BRS Louro & CEP 52 & BRS 177 & CEP 51 & Nova Era & BRS Tarumã \\
\hline $26 / 10 / 2005$ & $0,97 \mathrm{Dea}^{*}$ & $1,10 \mathrm{Ea}$ & $1,10 \mathrm{Aa}$ & $0,73 \mathrm{Ca}$ & $0,90 \mathrm{Ca}$ & $1,50 \mathrm{Aa}$ \\
$21 / 12 / 2005+$ & $1,25 \mathrm{DEbc}$ & $1,35 \mathrm{DEab}$ & $1,70 \mathrm{Aa}$ & $0,87 \mathrm{Cc}$ & $1,61 \mathrm{Bab}$ & $-^{+}$ \\
$6 / 2 / 2006$ & $3,35 \mathrm{Aa}$ & $2,88 \mathrm{Aab}$ & $1,96 \mathrm{Ac}$ & $3,06 \mathrm{Aab}$ & $2,53 \mathrm{Abc}$ & $0,92 \mathrm{Ad}$ \\
$21 / 3 / 2006$ & $2,16 \mathrm{BCbc}$ & $2,39 \mathrm{Bab}$ & $1,59 \mathrm{Ac}$ & $2,93 \mathrm{Aa}$ & $2,09 \mathrm{ABbc}$ & $2,39 \mathrm{Aab}$ \\
$12 / 5 / 2006$ & $1,59 \mathrm{CDb}$ & $1,66 \mathrm{CDb}$ & $1,56 \mathrm{Ab}$ & $2,83 \mathrm{Aa}$ & $1,89 \mathrm{ABb}$ & $1,80 \mathrm{Ab}$ \\
$4 / 9 / 2006+\dagger \#$ & $1,38 \mathrm{DE} \dagger$ & $1,18 \mathrm{E} \dagger$ & $1,30 \mathrm{~A} \dagger$ & $1,42 \mathrm{~B} \dagger$ & $-{ }^{+}$ & $-{ }^{+}$ \\
$25 / 10 / 2006+\dagger$ & $0,79 \mathrm{E} \dagger$ & $1,12 \mathrm{E} \dagger$ & $1,44 \mathrm{~A} \dagger$ & $1,32 \mathrm{~B} \dagger$ & $2,05 \mathrm{AB} \dagger$ & $-{ }^{+}$ \\
$18 / 4 / 2007$ & $2,76 \mathrm{ABa}$ & $2,05 \mathrm{BCab}$ & $2,08 \mathrm{Aab}$ & $1,46 \mathrm{Bb}$ & $1,95 \mathrm{ABb}$ & $1,73 \mathrm{Ab}$ \\
\hline
\end{tabular}

* Médias seguidas por mesma letra maiúscula nas colunas e minúscula nas linhas não diferem entre si pelo Teste Tukey a 5\% de erro. +Plantas não atingiram o estágio de duplo anel e de espigueta terminal. "Não foi realizada a análise estatística na interação cultivar dentro das datas de semeadura pois apenas uma repetição. "Não foi possível fazer a determinação porque não foi medido o Estágio de Haun

trigo ocorrem em momentos distintos do desenvolvimento foliar em função do genótipo e do ambiente (datas de semeadura). Assim, práticas de manejo associadas com estes dois momentos, e que atualmente são baseados nos indicadores morfológicos início do perfilhamento e início da elongação do colmo (REUNIÃ̃O DA COMISSÃO BRASILEIRA DE PESQUISA DE TRIGO, 2008), podem ser melhoradas se o indicador morfológico for o número de folhas, representada pelo HS. Por exemplo, na data de semeadura de 06/02/2006 a cultivar BRS Louro teve $\mathrm{HS}_{\mathrm{IP}}=5,10$ e $\mathrm{HS}_{\mathrm{DA}}=7,13$, ou seja, diferença de duas folhas, que foi ainda maior na cultivar BRS Tarumã (13 folhas), pois o $\mathrm{HS}_{\mathrm{IP}}$ foi 4,89 (Tabela 4) e o $\mathrm{HS}_{\mathrm{DA}}$ foi 18,13 (Tabela 3). Já o início da elongação do colmo não foi medido neste experimento, mas geralmente ocorre alguns dias após a ET (STRECK et al., 2003a). Por outro lado, 
Tabela 7 - Diferença entre o número de folhas final de folhas (NFF) e o Estágio de Haun na espigueta terminal [NFF - $\left.\left(\mathrm{HS}_{\mathrm{ET}}\right)\right]$ em seis cultivares de trigo em doze datas de semeadura em cultivo em Santa Maria, RS, Brasil, entre 2005 e 2007. Santa Maria, RS, 2011

\begin{tabular}{|c|c|c|c|c|c|c|}
\hline \multirow{2}{*}{ Datas de semeadura } & \multicolumn{6}{|c|}{ Cultivares } \\
\hline & BRS Louro & CEP 52 & BRS 177 & CEP 51 & Nova Era & BRS Tarumã \\
\hline 24/6/2005\# & $1,90 \mathrm{Ab}^{*}$ & $2,35 \mathrm{DEa}$ & _\# & $2,37 \mathrm{BCa}$ & _\# & _\# \\
\hline $4 / 8 / 2005$ & $1,89 \mathrm{Ac}$ & 2,01 Ebc & 2,49 ABabc & $3,08 \mathrm{BC}$ & 3,07 CDa & 2,75 ABab \\
\hline 7/9/2005+ & $1,91 \mathrm{Ab}$ & 2,71 CDEa & 2,23 ABab & $1,94 \mathrm{Cb}$ & 2,44 CDab & ${ }_{-}^{+}$ \\
\hline 26/10/2005 & 2,13 Acd & 3,32 BCDbc & 2,97 ABbc & $3,62 \mathrm{Bb}$ & $5,03 \mathrm{ABa}$ & $1,08 \mathrm{Cd}$ \\
\hline $21 / 12 / 2005+$ & 3,07 Aa & 3,63 $\mathrm{BCa}$ & $2,74 \mathrm{ABa}$ & $3,50 \mathrm{Ba}$ & 3,77 BCDa & $-^{+}$ \\
\hline $6 / 2 / 2006$ & $1,89 \mathrm{Ad}$ & $5,14 \mathrm{Aa}$ & $2,70 \mathrm{ABcd}$ & 3,74 Bbc & $4,22 \mathrm{ABCab}$ & $1,44 \mathrm{BCd}$ \\
\hline $21 / 3 / 2006$ & $2,34 \mathrm{Ab}$ & $3,94 \mathrm{Ba}$ & 3,11 Aab & $3,05 \mathrm{BCab}$ & 3,79 ABCDa & 2,99 Aab \\
\hline $12 / 5 / 2006$ & 2,58 Aa & 2,86 CDEa & $2,77 \mathrm{Aa}$ & 2,60 BCa & 2,81 CDa & 3,31 Aa \\
\hline $4 / 9 / 2006+\dagger$ & $3,12 \mathrm{~A} \dagger$ & $3,27 \mathrm{BCD} \dagger$ & $2,03 \mathrm{~B} \dagger$ & $2,35 \mathrm{BC} \dagger$ & $2,15 \mathrm{D} \dagger$ & ${ }_{-}^{+}$ \\
\hline $25 / 10 / 2006+\dagger$ & $2,49 \mathrm{~A} \dagger$ & $4,05 \mathrm{~B} \dagger$ & $2,80 \mathrm{AB} \dagger$ & $3,47 \mathrm{~B} \dagger$ & $2,47 \mathrm{CD} \dagger$ & $-^{+}$ \\
\hline $18 / 4 / 2007$ & $2,45 \mathrm{Aa}$ & 3,17 BCDa & $2,84 \mathrm{Aba}$ & $3,30 \mathrm{BCa}$ & $3,18 \mathrm{CDa}$ & $3,23 \mathrm{Aa}$ \\
\hline $7 / 6 / 2007 \dagger \#$ & _\# & _\# & _\# & $5,31 \mathrm{~A} \dagger$ & $5,58 \mathrm{~A} \dagger$ & _\# \\
\hline
\end{tabular}

* Médias seguidas por mesma letra maiúscula nas colunas e minúscula nas linhas não diferem entre si pelo Teste Tukey a 5\% de erro. ${ }^{+}$Plantas não atingiram o estágio de espigueta terminal. `Não foi realizada a análise estatística na interação cultivar dentro das datas de semeadura pois apenas uma repetição. "Não foi possível fazer a determinação porque não foi medido o Estágio de Haun

poder-se-ia argumentar que a medida do HS pode ser uma desvantagem a campo, já que exige mão de obra para fazer a medida do comprimento das duas folhas apicais e a contagem do número de folhas no colmo principal. Esta desvantagem pode ser superada com o uso de modelos matemáticos que simulam o aparecimento de folhas e o HS em nível diário. Entre esses modelos destacam-se aqueles mais simples, baseados no conceito do filocrono (STRECK et al., 2005), e aqueles mais complexos, que consideram a resposta não linear do aparecimento de folhas à temperatura, fotoperíodo e idade da planta (STRECK et al., 2003b; STRECK et al., 2007; XUE; WEISS; BIENZIGER, 2004).

\section{CONCLUSÕES}

1.O número de folhas no colmo principal, representado pelo Estágio de Haun, no duplo anel e na espigueta terminal em trigo varia com a cultivar (grupo de maturação) e com a data de semeadura, de modo que em cultivares precoces e em semeaduras nos meses mais frios o $\mathrm{HS}_{\mathrm{DA}}$ e $\mathrm{HS}_{\mathrm{ET}}$ são menores do que em cultivares tardias e em semeaduras nos meses mais quentes;

2.O indicador morfológico número de folhas representado pelo Estágio de Haun (HS) pode ser utilizado com o propósito de auxiliar em práticas de manejo da cultura do trigo.

\section{REFERÊNCIAS}

ALBERTO, C. M. et al. Resposta à vernalização de algumas cultivares brasileiras de trigo. Bragantia, v. 68 , n. 02, p. 535543, 2009a.

ALBERTO, C. M. et al. Modelagem do desenvolvimento de trigo considerando diferentes temperaturas cardinais e métodos de cálculo da função de resposta à temperatura. Pesquisa Agropecuária Brasileira, v. 44, n. 06, p. 545-553, 2009 b.

COMISSÃO DE QUÍMICA E FERTILIDADE DO SOLO. Manual de adubação e calagem para os estados do Rio Grande do Sul e Santa Catarina. 10. ed. Porto Alegre: Sociedade Brasileira de Ciência do Solo; Comissão de Química e Fertilidade do Solo, 2004. 400 p.

COMPANHIA NACIONAL DE ABASTECIMENTO. Safras - Grãos: Avaliação da Safra Agrícola 2007/2008 - Segundo Levantamento de Intenção de Plantio - Novembro/2007. Disponível em: < http://www.conab.gov.br/conabweb/download/ safra/estudo_safra.pdf $>$. Acesso em: 15 fev. 2009.

GOMES, F. P. Curso de estatística experimental. 13. ed. Piracicaba: Nobel, 1990. 468 p.

HAUN, J. R. Visual quantification of wheat development. Agronomy Journal, v. 65, n. 01, p. 116-119, 1973.

INSTITUTOBRASILEIRODEGEOGRAFIAEESTATÍSTICA.

Estatística da Produção Agrícola, 2011. Disponível em: <http:// www.ibge.gov.br>. Acesso em: 23 nov. 2011. 
KUINCHTNER, A.; BURIOL, G. A. Clima do Estado do Rio Grande do Sul segundo a classificação climática de Köppen e Thornthwaite. Disciplinarum Scientia, v. 02, n. 01, p. 171-182, 2001.

McMASTER, G. S. Phytomers, phyllochrons, phenology and temperate cereal development. Journal of Agricultural Science, v. 143, n. 02/03, p. 137-150, 2005.

MUNDSTOCK, C. M. Planejamento e manejo integrado da lavoura de trigo. 2. ed. Porto Alegre, 1999. 227 p.

PORTER, J. R. et al. An analysis morphological development stages in Avalon winter wheat crops with different sowing dates and at ten sites in England and Scotland. Journal of Agricultural Sciences, v. 109, p. 107-121, 1987.

REUNIÃO DA COMISSÃO BRASILEIRA DE PESQUISA DE TRIGO. Informações técnicas para a safra 2007: trigo e triticale. Passo Fundo: EMBRAPA TRIGO, 2006. 75 p.

REUNIÃO DA COMISSÃO BRASILEIRA DE PESQUISA DE TRIGO. Informações técnicas para a safra 2009: trigo e triticale. Passo Fundo: EMBRAPA TRIGO, 2008. 172 p.

RITCHIE, J. T. Wheat phases development. In: HANKS, R. J.; RITCHIE, J. T. (Ed.). Modeling Plant and Soil System. Madison: ASA, CSSA, SSSA, 1991. p. 31-54.

RODRIGUES, O. et al. Efeito da apliaçãode herbicida hormonal em diferentes estádios de desenvolvimento de trigo (Triticum aestivum L. Cvs. Embrapa 16 e BR 23). Revista Brasileira de Herbicidas, v. 05, n. 01, p. 19-29, 2006.

ROSA, H. T. et al. Métodos de soma térmica e datas de semeadura na determinação de filocrono de cultivares de trigo. Pesquisa Agropecuária Brasileira, v. 44, n. 11, p. 1374-1382, 2009.

SINCLAIR, T. R.; JAMIESON, P. D. Grain number, wheat yield, and bottling beer: na analysis. Field Crops Research, v. 98, n. 01 , p. $60-67,2006$.
STRECK, N. A. et al. Improving predictions of developmental stages in winter wheat: a modified Wang and Engel model. Agricultural and Forest Meteorology, v. 115, n. 03, p. 139-150, 2003a.

STRECK, N. A. et al. Incorporating a chronology response function into the prediction of leaf appearance rate in winter wheat. Annals of Botany, v. 92, n. 02, p. 181-190, 2003 b.

STRECK, N. A. et al. Estimativa do filocrono em cultivares de trigo de primavera. Revista Brasileira de Agrometeorologia, v. 13, p. 423-429, 2005.

STRECK, N. A. et al. Duração do ciclo de desenvolvimento de cultivares de arroz em função da emissão de folhas no colmo principal. Ciência Rural, v. 36, n. 04, p. 1086-1093, 2006.

STRECK, N. A. et al. Simulating leaf appearance in some Brazilian spring wheat genotypes. Revista Brasileira de Agrometeorologia, v. 15, p. 1-9, 2007.

STRECK, N.A. et al. Relationship between panicle differentiation and main stem leaf number in rice genotypes and red rice biotypes. Scientia Agrícola, v. 66, n. 02, p. 195-203, 2009.

UNITED STATES DEPARTMENT OF AGRICULTURE. Foreign Agricultural Service. Production, Supply and Distribution Online: Custom Query. USA, 2011. Disponível em: <http://www.fas.usda.gov/ >. Acesso em: 24 nov. 2011.

XUE, Q.; WEISS, A.; BAENZIGER, P. S. Predicting leaf appearance in field-grown winter wheat: evaluating linear and non-linear models. Ecological Modelling v. 175, n. 03, p. 261-270, 2004.

WALTER, L. C. et al. Desenvolvimento vegetativo e reprodutivo de cultivares de trigo e sua associação com a emissão de folhas. Ciência Rural, v. 39, n. 08, p. 2320-2326, 2009.

WENDT, W.; DUCA, L. J. L. Del; CAETANO, V. R. Avaliação de cultivares de trigo de duplo propósito, recomendados para cultivo no estado do Rio Grande do Sul. Pelotas: Embrapa Clima Temperado, 2006. 2 p. (Comunicado Técnico, 137). 